\title{
Distributed ISD Team Leadership and the Paradox of Cohesion and Conflict
}

\author{
Stephen McCarthy \\ Paidi O'Raghallaigh \\ Ciara Fitzgerald \\ Frédéric Adam \\ Cork Univ. Business School Cork Univ. Business School Cork Univ. Business School Cork Univ. Business School \\ University College Cork University College Cork University College Cork University College Cork

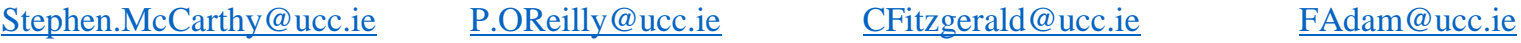

\begin{abstract}
Distributed ISD projects are often typified by deepseated differences between team members from diverse organizational and professional backgrounds. Consequently, literature suggests that cohesion is crucial for aligning the efforts of a distributed ISD team; however, a competing body of literature also asserts that conflict is essential for capitalizing on diverse knowledge flows. Team leaders can therefore face a conundrum around how to balance the paradoxical need for both cohesion and conflict. In this paper, we develop a theoretical framework to analyze case study findings from the 'CDSS project', a distributed ISD project undertaken in an Intensive Care Unit (ICU). We find evidence that distributed ISD leaders must adopt a 'paradox mindset', one which embraces both cohesion and conflict. Based on these findings, we also put forward the concept of 'leadership intelligence' which describes the simultaneous enactment of a diverse set of leadership styles for balancing constructive cohesion and conflict.
\end{abstract}

\section{Introduction}

Information System Development (ISD) is a crucial mechanism for modern organizations to respond to changes in the internal and external environment. However, the management of ISD is an inherently complex task. According to The Standish Group [1], 52\% of ISD projects in 2015 encountered significant challenges, while $19 \%$ were deemed to have failed. A significant body of literature has been dedicated to outlining the criteria for ISD project success; yet despite this, the rates of ISD project failure continue to remain high. IS scholars increasingly point towards the need to manage social aspects of ISD as it is a key determinant of ISD performance [2]. For instance, ISD team performance can be hampered due to a lack of cohesion owing to interpersonal differences between groups [3].

Distributed ISD projects are a unique category of ISD practice in which team members are organizationally, geographically, or temporally dispersed [4]. The creation of clear and agreed IT solutions is often inhibited in distributed ISD settings due to tensions between macro-level patterns and microlevel interactions among team members [5, 6]. For instance, macro-level differences between the positions, interests, and values of a distributed team in turn constrain and enable the interactions between team members during the development of an IT artefact.

While team cohesion is essential for the performance of distributed teams [7,8], there is also a competing body of literature which states that effective decision making in distributed settings requires conflict in order to capitalize on the diverse knowledge flows of multidisciplinary specialists $[9,10]$. In particular, ISD team leaders are presented with the problem of balancing the opportunities afforded by a divergence of ideas through conflict, while still aligning team members' efforts through sufficient levels of cohesion. This presents ISD team leaders with the significant challenge of understanding how to simultaneously address the paradoxical phenomena of cohesion and conflict. According to Quinn [11], leaders must enact different styles of leadership to address paradoxical tensions, utilizing their intuition and experience to move beyond planning alone $[12,14]$. Our ability to understand the role of leadership in balancing this paradox will be crucial for ensuring team effectiveness going forward.

According to Fairhurst, et al. [12], such paradoxes require new theoretical lenses which allow researchers and practitioners to both 'zoom in and zoom out' from the micro-level interactions and the contextual macrolevel patterns to better understand the emergence of paradoxes. However, ISD literature to date has yet to explore how the interplay of macro-level patterns and micro-level interactions impact cohesion and conflict in distributed teams. In addition, the role of leadership in balancing these paradoxical phenomena has yet to be 
explored. The research objective of this paper is to investigate the interplay between macro- and microlevel factors, cohesion and conflict, and the leadership of distributed ISD teams. Based on this objective, we investigate the following research question: What is the role of different leadership styles in dealing with cohesion and conflict in distributed ISD teams? Empirical findings are gathered from the in-depth case study of a Clinical Decision Support System (CDSS) project in order to explore and provide insights. The case study was conducted over a five-month timeframe, during which the distributed ISD project team faced acute challenges when designing a decision support system for the mission critical environment of an ICU. We develop a theoretical framework to describe and explain interactions among the distributed team and investigate the factors that affect cohesion and conflict.

The remainder of the paper is structured as follows: Section 2 reviews relevant literature published between 2000 and 2018 in the AIS senior scholar basket of eight journals and prominent IS conferences. Section 3 introduces the research design while Section 4 develops the theoretical framework. Section 5 presents findings from the case and Section 6 discusses these findings as relevant to academic and practitioner communities. Section 7 offers a conclusion.

\section{Literature Review}

ISD projects are an innately social undertaking as individuals must continuously interact to share ideas, resolve differences, and coordinate resources [13, 14]. For instance, ISD projects typically involve participants from diverse backgrounds who engage in an emergent process of communication, sense-making and negotiation around the proposed system $[15,16]$. Some scholars argue that IS primarily concerns the social construction of knowledge, where individuals and groups seek to collaboratively build new understandings while developing a system [13, 15, 17]. Accordingly, individuals engage in social interactions to share and integrate the knowledge required for systems development within a set timeframe $[13,17]$.

ISD projects are increasingly conducted by distributed teams consisting of individuals from different organizational, geographic, and disciplinary backgrounds [2, 6, 8]. Distributed ISD project teams must collaborate remotely across different locations and often across different time zones in order to perform tasks. This is facilitated by the advent of increasingly sophisticated IT solutions such as email, instant messaging, and video conferencing [2, 5]. However, despite these advances, distributed project teams still face inherent challenges around collaboration [5, 6, 7]. Previous IS studies therefore suggests that team cohesion is a key determinant of team performance in distributed ISD projects [7, 9, 10].

Team cohesion can be defined as the extent to which team members are aligned in their shared understanding of and shared commitment to project tasks e.g. the actions that individuals and groups need to perform based on agreed plans [10]. Shared understanding and shared commitment are essential for cohesion in diverse teams [10]. They also help ensure the durability of solutions designed for tackling identified problems [18]. Shared understanding refers to "the degree to which people concur on the value of properties, the interpretation of concepts, and the mental models of cause and effect with respect to an object of understanding" [19, pg. 115]. Shared commitment then refers to the degree to which team members are willing to dedicate resources towards the delivery of proposals that have gained shared understanding [10, 18, 20].

However, generating cohesion in distributed ISD teams is an inherently challenging task for leaders due to interpersonal differences between individuals and groups [10]. Literature points towards challenges that can arise between 'subgroups' in distributed ISD teams characterized by diverse disciplinary backgrounds, skill sets, experience etc. [21, 22]. Subgroups can form where team members perceive hypothetical divisions, also referred to as 'faultlines', between other members of the project team [23, 24]. As stated by Carton and Cummings [21], the co-existence of subgroups creates a notable change to the team dynamic as subgroup members must continuously remain cognizant of subgroup members as well as other subgroups. Subgroups can develop fragmented interests and meanings around the problem-solution coupling which creates challenges in identifying a way forward.

While cohesion is recognized by IS scholars as an important determinant of team performance, there is also a body of literature which points towards the negative impact of excessive cohesion among project teams [cf. 9, 25]. For instance, McAvoy and Butler [9] suggests that excessive levels of cohesion can impede the performance of ISD project teams where the drive for consensus inadvertently suppresses disagreement and the appraisal of alternatives. This can have a negative impact on project outcomes, as the suppression of divergent ideas can limit the development of innovative and effective IT artefacts [9, 22]. Team conflict can be defined as the extent to which team members diverge in their shared understanding of and shared commitment to project tasks [9]. Studies have shown that team conflict can improve team performance as it promotes the critical analysis of project tasks [3].

Literature differentiates between conflict which is 'constructive' and 'destructive' to team performance. Constructive conflict occurs when team members deal 
with differences in interpretation around tasks through argumentation and clarification [3, 26]. Meanwhile, destructive conflict centers on social differences between team members in terms of their positions, interests, values. Similarly, cohesion can be categorized as constructive and destructive in nature. Constructive cohesion helps align the efforts of team members through shared understanding and shared commitment, while destructive cohesion can emerge where the appraisal of alternatives is suppressed due to groupthink among members of the team [9].

A key challenge for ISD team leaders therefore centers on how best to balance the opportunities afforded by constructive conflict, while still maintaining sufficient levels of cohesion. Quinn [11] suggests that in order to address organizational paradoxes, team leaders must enact different leadership styles that foster both stability and flexibility (see Table 1). Wakefield et al. [7] found that three of these styles outlined by Quinn [11] mitigate conflict, whereas there was no conclusive evidence that the fourth style (mentor) had a direct impact on conflict. However, qe find that both Quinn [11] and Wakefield et al. [7] fail to consider constructive conflict for organizational and team performance. Therefore, it remains unexplored whether these styles are sufficient to balance both cohesion and conflict.

Table 1: Styles of Team Leadership (after [11])

\begin{tabular}{|c|l|}
\hline Style & \multicolumn{1}{|c|}{ Description } \\
\hline Coordinator & $\begin{array}{l}\text { Maintains stability by setting rules } \\
\text { and standards, and outlining } \\
\text { constraints. A coordinator style aims } \\
\text { to control the team's assigned work. }\end{array}$ \\
\hline Monitor & $\begin{array}{l}\text { Creates stability by measuring } \\
\text { progress, and distributing this data. A } \\
\text { monitor style aims to oversee the } \\
\text { work that the team must accomplish. }\end{array}$ \\
\hline Facilitator & $\begin{array}{l}\text { Fosters flexibility by seeking } \\
\text { consensus around divergent opinions. } \\
\text { A facilitator aims to actively listen to, } \\
\text { and negotiate team differences. }\end{array}$ \\
\hline Mentor & $\begin{array}{l}\text { Promotes flexibility by supporting the } \\
\text { personal development of individuals. } \\
\text { A mentor style aim to create } \\
\text { awareness of team members' needs. }\end{array}$ \\
\hline
\end{tabular}

\section{Research Design}

An in-depth case study approach [cf. 27] was chosen to study the information-rich case of a distributed ISD project. This was selected as the most appropriate research design as it enables the researcher to elicit detailed accounts of individuals' actions, experiences, and perspectives in their natural setting. The project in question, the CDSS project, had two main objectives: the development of software to support decision making in the ICU ward, and the conduction of a research study to evaluate this solution for improving patient outcomes.

The ISD project team consisted of a team leader and two subgroups: the 'clinical subgroup' consisting of a ICU dietician, clinical lead, and pharmacist; the R\&D subgroup consisting of the developer, postdoctoral researcher, research officer, and research nutritionist. The ISD project team was distributed across three locations: a public hospital, the main campus of a university, and a research center located off-site in a satellite campus. The project team utilized IT solutions such as email, conference calls, and an online knowledge repository. Subject to the availability of team members and their ability to travel to the research center, face-to-face meetings were also organized.

The case study focuses on a five-month timeframe between November 2016 and March 2017. The lead author was located in the research center (two to three days a week, eight hours a day). In addition, the lead author attended team meetings (each typically lasting 2 hours), and regular meetings with individual team members around work progress and challenges. To increase robustness of findings, case study data was triangulated from three different sources [cf. 28]. (i) The lead author recorded 51 pages of participant observations in field notes. (ii) This data was complemented by eight semi-structured interviews conducted with members of the team between June and October 2017. Each face-to-face interview lasted between 45 and 60 minutes and was recorded and transcribed. (iii) Project documents were collected and analyzed to unearth further insights. This included over 70 team emails, 14 slide decks, and 11 documented meeting minutes.

The authors then developed an evolving theoretical framework [5, 29] (outlined in Section 4) which set out the initial research themes. The framework was iteratively reviewed and refined through reflection on and analysis of the collected data [cf. 30]. The lead author analyzed the case study data from November 2017 onward using two primary techniques: coding and vignettes. Open, axial, and selective coding (as per Strauss and Corbin [31]) were used to analyze the transcribed interview data. The lead author's perception of variables and relationships, otherwise referred to as theoretical sensitivity, was influenced by the theoretical development. Initially, the lead author coded 27 nodes in NVivo, and then aggregated these into 9 overarching nodes. Finally, selective coding was completed using the theoretical framework. Vignettes as per Miles and Huberman [28] were also used to produce, reflect on, and learn from participant observation data and key moments in the 'everyday life' of the project. In addition, the lead author met weekly with co-authors to 
recount his observations and make sense of findings. During these meetings, which typically lasted one to two hours, the other authors would question the lead author about the data in order to extract relevant themes.

\section{Theoretical Development}

In investigating the research question, the authors developed a theoretical framework to assist in describing and explaining how the interplay between the macro-level patterns and micro-level interactions impacts cohesion and conflict in distributed ISD project teams. The macro-level relates to those large-scale social patterns and trends which shape individual behaviors overtime, whereas the micro-level concerns the study of interactions between individuals and objects in the field [6]. The term interplay refers to the reciprocal relationship between the two dimensions which exist at different levels of analysis i.e. macro and micro. For instance, micro-level interactions may produce patterns which eventually become established as macro-level constructs. These macro-level constructs then both constrain and enable team interactions.

Theory building was undertaken following the structured-case approach [cf. 30, pg. 236] which consists of "constructing and articulating a preliminary conceptual structure, collecting and analyzing data, and reflecting on the outcomes to build knowledge and theory". The resulting framework is grounded in both $a$ priori concepts from existing literature and a posteriori insights from the case study. The authors first drew on a priori macro- and micro-level concepts from the seminal works of Parsons [32] and Bourdieu [33]. A posteriori empirical data was then used to examine the interplay between these macro- and micro-level concepts, and how the interplay impacts cohesion and conflict.

Building on Parsons [32], our framework looks at three macro-level factors: Structure, Identity, and Culture. Structure deals with the different positions, roles, and rules which shape how team members take action across situations. Identity deals with the different interests of team members which motivate their courses of action. Finally, Culture refers to the different shared meanings, values, and assumptions which are internalized by team members.

Building on Bourdieu [33], we turn attention to three micro-level factors: Vision, Approach, and Means. The construct of Vision deals with the intended course of action which will be pursued by individuals in the field of practice, and which in turn shapes their decisions and utilization of resources in the field. Approach refer to the 'modus operandi' of how individuals achieve a vision which is guided by the tacit knowledge acquired through their accumulated experience in practice.
Means refers to the resources or forms of capital which are utilized by individuals to pursue visions in the field.

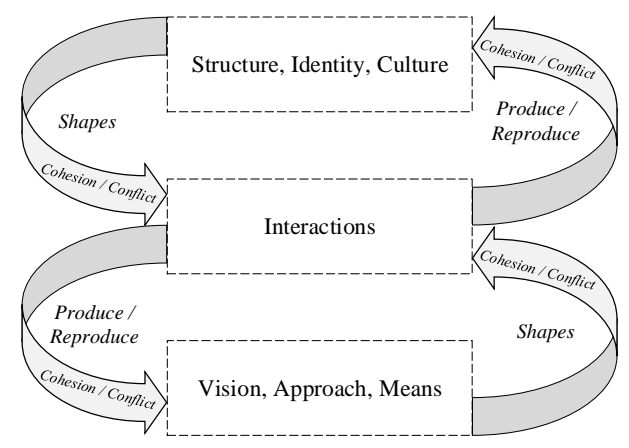

Figure 1. Conceptual Diagram

Figure 1 combines the theoretical pillars to illustrate how this interplay impacts cohesion and conflict between subgroups and the team leader. The upper half of the diagram illustrates how structure, identity and culture shape interactions, and how these interactions in turn produce and reproduce the macro-level. The lower half of the diagram shows how interactions produce and reproduce the vision, approach, and means, which further shape interactions. While authors such as Pettigrew [34] have previously looked at context and process interactions within an organizational setting, our theoretical framework is differentiated by its specific focus on how the interplay between macro- (i.e. structure, identity culture) and micro-level (i.e. vision, approach, means) factors shape the paradoxical tension between conflict and cohesion in distributed ISD teams.

\section{Findings}

This section discusses how the interplay between the macro- and micro-level impacted cohesion and conflict between the team. The subsections describe three examples based on cells of the framework which best demonstrate the paradox of cohesion and conflict.

\subsection{Interplay between Structure and Vision}

During recruitment, the team leader had briefed each individual on what the project would entail; however, the exact structure of the distributed ISD team was not defined upfront. Team members recognized that the team leader was at the apex of one hierarchy for decisions relating to the project and the research study, while the clinical lead was at the apex for decisions relating to the software and its implementation in the ICU ward. Meanwhile, the position of other team members resembled a flat hierarchy.

However, in performing their work, individuals began to position themselves against an evolving team 
hierarchy. In this de-facto hierarchy, the ICU dietician assumed a more prominent position and asserted her own vision for the research study and software solution. At the same time, the developer was relegated to a low position in the hierarchy as other team members saw his role as being of secondary importance to the project. As a result, the developer's vision for the software was oftentimes less influential in the team interactions. Reinforcing this de-facto hierarchy, some team members began to utilize private email interactions and side meetings to expedite decision-making. For instance, some decisions around the research study took place during private meetings between the team leader, ICU dietician, and the research nutritionist. This was constructive initially as it enabled some team members to clarify ambiguities around the emerging vision. This emerging vision in turn shaped the subgroup interactions as the discussion began to center on the impediments to these visions.

Individuals who were not included in these meetings did not have visibility of ongoing discussions, despite the pertinence of their input, which the pharmacist felt was problematic: "you can feel a bit excluded from parts of the project if you hear 'oh they're meeting today, ok I'm not involved in that'. I think it's not good for the communication in the project". This impeded cohesion and led to fragmented discussions around the vision as some team members did not have oversight on decisions. In addition, the roles of team members sometimes seemed to overlap which made it difficult to resolve conflict around the vision, such as in the case of the ICU dietician and research nutritionist. As stated by the postdoctoral researcher, the ICU dietician and research nutritionist both assumed they had the final say on the revised ICU guidelines which created: "some confusion in the project between the ICU dietician and research nutritionist". As a result, the de-facto hierarchy eventually collapsed due to uncertainty around who had the final say on decisions, and this in turn led to increasing levels of conflict around the vision of the project. The developer began to disagree with the team leader's decisions and tried to assert his position by assuming responsibility for deadline setting and repeatedly called on team members to provide feedback on the software's requirements. However, no action was taken by others in the team as he was seen as only having an operational role in the project.

\subsection{Interplay between Identity and Means}

Delineations between the professional identities of team members in turn shaped interactions during meetings. These delineations were created by the team leader to assert the domain expertise of team members during discussions around the project. For instance, the team leader drew delineations between team members who were identified as "scientists" and "non-scientists" based on whether or not they had the means to conduct research. The team leader observed that: "clinicians aren't scientists and they needed to learn how to conduct science from scientists. On the other side, scientists aren't clinicians". The clinical subgroup was also quick to delineate between the expertise of team members who were identified as "clinical" and "nonclinical", based on whether they had working knowledge of the daily practices in the ICU ward. These delineations were constructive and helped team members figure out who to direct specific questions to.

However, based on these delineations, the developer found himself with the challenging professional identity of a 'middle man' between two disciplines, as he was neither a 'clinician' nor a 'scientist'. As the sole IT expert on the team, the developer felt he didn't have the means to deliver on all that was being asked of him and referred to his predicament as "a team of one". Cohesion suffered as other team members saw the developer's professional identity as separate from the rest of the team. The developer tried to challenge this identity during interactions by requesting feedback however, other team members did not recognize his means to enact change. Over time the developer became increasingly isolated, eventually distancing himself from the project. The team leader also conceded that she often had limited knowledge of the work that the developer had completed which meant that "there has to be massive trust; that's really problematic for me".

Differences in team members' professional interest also emerged within subgroups, such as in the case of the clinical lead and ICU dietician. At the second project meeting, the clinical lead had outlined his professional interest in ensuring that the project should not generate disruptive change in the ICU ward. Based on this, he proposed that the software solution would only display digitalized patient information and consequently, any additional feature including the predictive modelling of patient outcomes would be ruled out of scope. Because of his senior position in the hospital, the clinical lead was able to enforce this decision and generate team cohesion around the scope. However, following this meeting, the clinical lead's engagement in the project temporarily ceased for the subsequent four months of the project, and the ICU dietician's professional interests became more influential in discussions around the software. For instance, the ICU dietician began to insist that the software solution should include a predictive modelling feature to support decision making which contradicted the clinical lead's original decision. The ICU dietician noted her vested professional interest in this feature: "I think that it will strengthen the role of nutrition in the unit... Information is power and I think 
that it will be very useful". This conflict around the scope helped open up discussions around how the software would differentiate itself from existing technology platforms in the ICU ward. Nevertheless, members of the R\&D subgroup were concerned that the clinical lead would later veto the ICU dietician's decisions once he became aware of it. Eventually the team leader facilitated a meeting between the clinical lead and ICU dietician, where the clinical lead decided to concede that the predictive modelling should be ruled in scope. However, uncertainty remained among the R\&D subgroup around whether this question was fully resolved. For example, the developer suspected that the clinical lead was not fully convinced of the benefits associated with the modelling feature. The developer questioned whether the clinical lead might yet reverse this decision later on, forcing considerable rework.

\subsection{Interplay between Culture and Approach}

The value placed on flexibility and exploratory discussions by the team leader shaped interactions between team members. For instance, the team leader deferred the creation of a project plan, and often dropped items from the meeting agenda to allow more time for dialogue. This approach was beneficial at the start of the project as it facilitated learning and constructive conflict around what the software should achieve. The leader afforded team members the opportunity to question disciplinary experts on the team and learn about what their work involved. In addition, the leader dropped less important items from the agenda and allowed team members to focus on discussion around the value proposition of the software for users in the ICU ward.

However, subgroup members felt that this approach created uncertainties around the interdependencies between team members' tasks and the critical path of the project. As stated by the pharmacist: "(we needed) a project plan to work towards... and someone following up to say 'this is your role, have you done it?". The R\&D subgroup requested clarifications from the team leader on how work should proceed. However, this created bottlenecks in the decision-making process as the team leader was not always available to respond in a timely fashion. As a result, the developer, for one, aired his concern that development work would take longer than expected, due to the challenges faced in sharing an understanding of requirements. The developer noted: "The project is essentially managing itself which is a problem... I'm the only one putting up the deadlines".
Each subgroup came with different cultural assumptions around the level of complexity involved in the project which also shaped interactions with the team leader. The ICU dietician assumed that her prior PhD research had specified the software's data requirements. However, the developer did not share this viewpoint and instead he felt that the detail around requirements had yet to be determined. As stated by the developer: "The problem is that clinicians think that the requirements are already packaged... They assume that we already have requirements - the short answer is no". In order to challenge cultural assumptions, the developer adapted his approach by sending repeated emails directly to the team leader and clinical subgroup which pointed to areas where clarification was needed. Eventually this led to high levels of conflict as team members became frustrated with the developer's preoccupation with uncertainties. As stated by the team leader: "I don't know if this is an individual thing or a discipline issue but (the developer's) tendency is always to see the pitfalls before anything else is even acknowledged". The developer challenged the clinical subgroup by pointing out shortcomings in their thinking but most team members seemed unaware that the developer was doing this in order to elicit software requirements.

\section{Discussion}

Extant literature on distributed ISD teams has primarily focused either on the micro-level interactions between team members, or on the contextual macrolevel patterns that tend to persist over time [6]. However, such a dualist perspective can limit understanding of how micro-level interactions shape macro-level patterns and vice versa. The theoretical framework developed by the authors was used to examine how the interplay between the macro and micro-level impacts cohesion and conflict in the CDSS project. Table 3 provides a summary of the findings discussed in section 5. The findings point towards how the interplay between macro-level patterns and microlevel interactions shaped the conduct of the distributed ISD project, and in turn impacted cohesion and conflict. It should be noted that findings from a single case are unlikely to be generalizable to all settings [27]. Nevertheless, in this section, we seek to put forward a set of propositions based on our case study findings which can be examined in future studies.

Table 3. Typology for Organizational ISD Practice Findings

\begin{tabular}{|c|l|l|l|}
\cline { 2 - 4 } \multicolumn{1}{c|}{} & \multicolumn{1}{c|}{ Structure } & \multicolumn{1}{c|}{ Identity } & \multicolumn{1}{c|}{ Culture } \\
\hline Vision & $\begin{array}{l}\text { The team leader's flat hierarchy } \\
\text { helped clarify ambiguities }\end{array}$ & $\begin{array}{l}\text { The team leader embraced } \\
\text { conflicting interests within the }\end{array}$ & $\begin{array}{l}\text { The team leader's openness to } \\
\text { conflicting assumptions around }\end{array}$ \\
\hline
\end{tabular}




\begin{tabular}{|c|c|c|c|}
\hline & $\begin{array}{l}\text { around the vision through } \\
\text { conflict. However, this } \\
\text { excessively inhibited cohesion } \\
\text { due to uncertainty around roles. }\end{array}$ & $\begin{array}{l}\text { clinical subgroup to clarify the } \\
\text { project vision. It took time to } \\
\text { resolve this conflict however } \\
\text { which inhibited cohesion. }\end{array}$ & $\begin{array}{l}\text { the vision helped clarify the } \\
\text { value proposition. However, } \\
\text { different assumption eventually } \\
\text { inhibited cohesion }\end{array}$ \\
\hline Approach & $\begin{array}{l}\text { The team leader endorsed } \\
\text { communication backchannels to } \\
\text { improve cohesion around the } \\
\text { approach. However, conflict } \\
\text { emerged as some members felt } \\
\text { excluded from these dialogs. }\end{array}$ & $\begin{array}{l}\text { The team leader identified the } \\
\text { developer as the sole IT expert } \\
\text { in the team which allowed him } \\
\text { to control the ISD approach. } \\
\text { However, this siloed approach } \\
\text { eventually inhibited cohesion. }\end{array}$ & $\begin{array}{l}\text { The value placed on flexibility } \\
\text { by the team leader enabled } \\
\text { learning and conflict. However, } \\
\text { other team members valued a } \\
\text { regimented approach which } \\
\text { eventually inhibited cohesion. }\end{array}$ \\
\hline Means & $\begin{array}{l}\text { The leader recognized that the } \\
\text { clinical subgroup's involvement } \\
\text { was crucial to cohesion around } \\
\text { the software requirements. } \\
\text { However, constrained input } \\
\text { from the clinical subgroup led to } \\
\text { conflict between team members. }\end{array}$ & $\begin{array}{l}\text { The team leader's delineations } \\
\text { between professional identities } \\
\text { generated cohesion by } \\
\text { clarifying domain expertise. } \\
\text { However, some team members } \\
\text { could not challenge their } \\
\text { identity which led to conflict. }\end{array}$ & $\begin{array}{l}\text { The leader's ability to foster } \\
\text { conflict around individuals' } \\
\text { diverse meanings helped } \\
\text { generate creative solutions. } \\
\text { However, this also inhibited } \\
\text { cohesion due to gaps in each } \\
\text { team members' knowledge. }\end{array}$ \\
\hline
\end{tabular}

Findings point to the paradoxical need for both cohesion and conflict in distributed ISD. For instance, the CDSS project highlights the inherent difficulties that can arise when distributed team leaders do not embrace the paradox of cohesion and conflict, and instead promote one element over the other. For instance, the style of leadership adopted by the team leader in the CDSS project primarily fostered conflict over cohesion which in turn impeded team performance. While the team leader's style initially helped promote exploratory dialogue, learning and creativity, the lack of coordination resulted in increasing levels of conflict and impeded cohesion. High levels of conflict arose between the developer and other team members around the vision of the project, and the overall approach.

However, a leadership style aimed at only promoting cohesion over conflict may also be ineffective. For instance, findings from our previous case study [35] suggest that a leadership style which prioritizes cohesion in all team interactions, and intentionally overly constrains the level of conflict, can impede the team's ability to challenge assumptions. Taken together, this suggests that distributed ISD team performance rests on balancing both cohesion and conflict.

Miron-Spektor, et al. [36] have pointed to the need for organizations to adopt a 'paradox mindset' which is both accepting of and energized by paradoxical tensions. However, the notion of a paradox mindset has not previously been applied to cohesion and conflict in distributed ISD teams. Building on our theoretical framework, we suggest that a paradox mindset in distributed ISD must cultivate a cognitive awareness of how the interplay between macro- and micro-level factors shapes cohesion and conflict. For instance, a paradox mindset might seek a balance between topdown structures and an emerging hierarchy a collective identity and individualized interests, and a single integrated culture and diverse cultures. We therefore put forward our first proposition which can be examined by future researchers and practitioners:

Proposition 1: The absence of a 'paradox mindset' [cf. 36] can lead to destructive cohesion and / or conflict in complex distributed ISD projects.

Our next proposition centers on team leadership styles in distributed ISD. Wakefield et al. [37] suggest that Quinn's [11] four team leadership styles are best suited to resolving different forms of conflict in distributed teams. However, Wakefield et al.'s [37] application of Quinn's [11] Competing Values Frameworks fails to consider the paradoxical tension between both cohesion and conflict in distributed ISD project teams. The authors discuss how the four leadership styles can be used to mitigate conflict, but do not reflect on the potential benefits of conflict such as creative problem solving and the avoidance of groupthink [9]. A paradox mindset must also recognize the importance of promoting conflict for team performance. For instance, our case study findings suggest that conflict can help challenge team members' assumptions and promote creativity during meetings.

Based on this insight, we aim to go beyond the four styles originally outlined by Quinn [11] and Wakefield et al. [37] to purpose a new style which we call 'agitator'. This can simultaneously be enacted alongside the previously mentioned four team leadership styles, and seeks to embed conflict into interactions in order to challenge cultural assumptions, foster divergent interests, and overcome structural silos. In particular, this additional style can encourage team members to adopt the role of devil's advocate [cf. 9] to ask challenging questions through focused periods of conflict. In the CDSS project, the developer often 
played the role of devil's advocate by questioning the ICU dietician and pharmacist, and challenging the logic behind their decisions. However, the developer at times was not supported in this role by the team leader as it was seen as an impediment to progress. The devil's advocate role can be constructive for challenging decisions before they are considered valid. Having said that, if left unchecked it can also become destructive. Team leaders must therefore learn when it is appropriate to enact the devil's advocate role and when it is not. Based on this, we put forward a second proposition:

Proposition 2: An 'agitator' style can promote constructive conflict in distributed ISD projects, but can lead to destructive conflict if left uncontrolled.

Finally, we propose that team leaders must cultivate 'leadership intelligence' in order to effectively respond to the paradox of cohesion and conflict in distributed ISD. We define leadership intelligence as the ability to simultaneously enact a diverse set of leadership styles (i.e. coordinator, monitor, facilitator, mentor, and agitator); in particular, leaders must alternate between 'closed' leadership behaviours (i.e. coordinator, monitor) which place constraints on individuals' actions, and 'open' leadership behaviours (i.e. mentor, agitator) which empower individuals by limiting centralised control. This leads us to one final proposition for future research:

Proposition 3: Leadership intelligence is essential for simultaneously balancing the paradox of cohesion and conflict in complex distributed ISD projects.

In proposing 'leadership intelligence', we extend the works of Quinn [11] and Wakefield, et al. [37] by asserting that leaders must become mindful of when to promote and supress different leadership styles in order to balance the paradoxical tension between cohesion and conflict during distributed ISD team interactions. For instance, over the course of a meeting, the leader may enact different leadership styles in order to frame macro- and micro-level factors in different ways depending on what the situation demands and dynamics between individuals in the room. This requires the sensitivity to know when the saturation point of each style is reached based on the leader's experience.

Leadership intelligence also fosters an awareness of how the interplay between macro-level patterns and micro-level interactions shape an ISD project. Closed leadership behaviors can aim to enforce deterministic macro-level patterns such as structure, identity, and culture to create constraints around team members' actions. For instance, leaders can enforce a clear topdown structure, and collective project-level identity and culture. Meanwhile, open leadership behaviors can seek to provide team members with the freedom to make decisions around the vision, approach, and means of practice. Leaders must alternate between these paradoxical leadership behaviors as circumstances demands. While leadership intelligence is also important for co-located teams, it becomes imperative in distributed ISD teams due to the unique challenges faced in these settings. For instance, the structure of a distributed team may not be clearly defined [6] which in turn can create uncertainty around the approach. In addition, the inherent diversity of distributed ISD teams can lead to differences in interests and culture meanings [7], which in turn leads to divergent perspectives.

Findings from the CDSS project suggest that the team leader did not recognise the switch from constructive to destructive cohesion and conflict. The team leader also did not effectively engage team members in necessary conversations around the vision, approach, and means of the project, and instead allowed unfocused conversations around team structures, identities, and cultures to continue. This led to periods of destructive conflict. While these discussions could have eventually been transformed into periods of constructive conflict, the team leader did not support the developer in enacting the role of devil's advocate and thus team members' positions, interests, and assumptions remained unchallenged, leading to continuing divisions.

While the findings suggest that the team leader did enact some leadership styles, these were not used effectively for balancing cohesion and conflict. Instead the team leader inadvertently enacted leadership styles at different points without recognising how they shaped both cohesion and conflict. This inadvertent use of leadership styles meant that sometimes the wrong style was enacted at the wrong time. For instance, the team leader at one point enacted a mentorship style to promote conflict around the team structure, despite calls from team members to enact a coordinator style and clarify the decision making hierarchy.

Leadership intelligence requires that team leaders enact different leadership styles simultaneously. For instance, a team leader could enact an agitator style to promote constructive conflict around the vision, while simultaneously enacting a coordinator style to promote constructive cohesion around the approach. Table 4 describes observations from the CDSS project on the aspects of leadership intelligence and provides recommendations around how team leaders can effectively balance cohesion and conflict through framing macro- and micro-level factors. However, as a whole the case study points to the need for leadership intelligence through its absence. 
Table 4: Aspects of Leadership Intelligence

\begin{tabular}{|c|c|c|}
\hline & Observations from CDSS Project & Recommendation \\
\hline 离 & $\begin{array}{l}\text { The level of coordination was limited but still } \\
\text { impeded constructive conflict as team members were } \\
\text { unclear about their roles and responsibilities. } \\
\text { Consequently, backchannels of communication } \\
\text { emerged in order to air differences around the vision. }\end{array}$ & $\begin{array}{l}\text { While the team leader did allow team members to air } \\
\text { their differences of opinion, ultimately she should } \\
\text { have provided further support to team members in } \\
\text { moving towards a shared understanding and } \\
\text { commitment to a vision and an approach. }\end{array}$ \\
\hline 营 & $\begin{array}{l}\text { Our findings show little evidence of a monitoring } \\
\text { style as exemplified by the lack of a formal project } \\
\text { plan. As a result, destructive conflict began to stifle } \\
\text { the progress of the project due to uncertainties around } \\
\text { the approach. }\end{array}$ & $\begin{array}{l}\text { While the team leader did place some value on a } \\
\text { flexible approach which provided team members with } \\
\text { an opportunity to engage in constructive conflict, she } \\
\text { should have addressed a shift towards destructive } \\
\text { conflict partially through more formalized planning. }\end{array}$ \\
\hline 莺 & $\begin{array}{l}\text { The facilitator style was adopted by the team leader } \\
\text { to help bridge the divergent interests of the ICU } \\
\text { dietician and clinical lead around the software } \\
\text { solution's vision; however, the absence of this style } \\
\text { later on created uncertainties around the vision. }\end{array}$ & $\begin{array}{l}\text { While the team leader did embrace some of the } \\
\text { divergent professional identities across the team, she } \\
\text { should have done this consistently and worked in } \\
\text { moving the different groups to a shared } \\
\text { understanding and commitment. }\end{array}$ \\
\hline 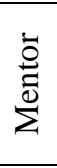 & $\begin{array}{l}\text { The team leader's style most resembled that of } \\
\text { mentorship in that it helped support team learning by } \\
\text { providing individuals with the flexibility needed to } \\
\text { explore the approach through discussion. }\end{array}$ & $\begin{array}{l}\text { While the team leader did foster a flexible culture } \\
\text { which allowed some exploratory dialogue, she should } \\
\text { have balanced this with a move towards a shared } \\
\text { understanding and commitment to a way forward. }\end{array}$ \\
\hline. & $\begin{array}{l}\text { Some team members did adopt the role of a devil's } \\
\text { advocate; however, the team leader showed little } \\
\text { acceptance of an agitator style as it was seen as an } \\
\text { impediment to progress. }\end{array}$ & $\begin{array}{l}\text { While the developer did adopt the role of devil's } \\
\text { advocate, the team leader should have supported and } \\
\text { placed more value on the benefits of this. }\end{array}$ \\
\hline
\end{tabular}

\section{Conclusion and Implications}

In this paper we sought to uncover how the interplay between macro- and micro-level factors impacts cohesion and conflict in the leadership of distributed ISD teams. We presented empirical findings from the case study of the CDSS project in order to derive insights into the leadership challenges emerging from the paradox of cohesion and conflict. From a theoretical perspective, this paper contributes a novel framework for describing and explaining ISD project team interactions within a distributed setting. The framework theorizes how the interplay between macro- (e.g. structure, identity, culture) and micro-level (e.g. vision, approach, means) factors impact team cohesion and conflict. This framework provides new theoretical perspectives on cohesion and conflict in distributed ISD.

From a practical perspective, the paper provides examples of the paradox of cohesion and conflict in action. While at face value, ISD projects may seem relatively straightforward, 'wickedness' [cf. 5] in the form of interpersonal differences between team members can create numerous challenges. For instance, the findings point towards the benefits of de-facto hierarchies for building cohesion around a vision but equally points to the challenges this creates in resolving conflict (Structure - Vision). Delineations between professional identities within a distributed team can also stimulate cohesion by clarifying domain expertise but may breed conflict where only some members have the means to enact change (Identity - Means). The value placed on a flexible approach can create opportunities to conflict but may eventually impede cohesion if there is limited levels of coordination (Culture - Approach).

Based on our findings, we set out three propositions for future researchers and practitioners. We firstly suggest that distributed teams may require a new type of team leader, one with a 'paradox mindset' [cf. 36] who understands how to shape macro- and micro-level factors so as to balance cohesion and conflict. We also put forward the concept of 'leadership intelligence' which sets out five different styles of leadership (i.e. coordinator, monitor, facilitator, mentor, agitator) for balancing cohesion and conflict.

One limitation of the case study is that the findings may not necessarily be generalizable to other contexts. Future research could examine the emergence of cohesion and conflict in distributed ISD teams that do not have a formal leadership role and the impact this has for the interplay of macro- and micro-level factors.

\section{References}

[1] The Standish Group, CHAOS Report, The Standish Group International, Boston, 2015. 
[2] J. Kotlarsky and I. Oshri, "Social ties, knowledge sharing and successful collaboration in globally distributed system development projects", European Journal of Information Systems, 14 (2005), pp. 37-48.

[3] T. Carte and L. Chidambaram, "A capabilities-based theory of technology deployment in diverse teams: Leapfrogging the pitfalls of diversity and leveraging its potential with collaborative technology", Journal of the Association for Information Systems, 5 (2004), pp. 4. [4] J. B. Windeler, L. M. Maruping, L. P. Robert and C. K. Riemenschneider, "E-profiles, conflict, and shared understanding in distributed teams", Journal of the Association for Information Systems, 16 (2015), pp. 608. [5] S. McCarthy, P. O'Raghallaigh, C. Fitzgerald and F. Adam, Exploring the Nuances of 'Wickedness' in Information Systems Development, HICSS-51 Proceedings, 2018. [6] S. Sarker and S. Sahay, "Understanding virtual team development: An interpretive study", Journal of the association for information systems, 4 (2003), pp. 1. [7] G. Garrison, R. L. Wakefield, X. Xu and S. H. Kim, "Globally distributed teams: The effect of diversity on trust, cohesion and individual performance", The DATABASE for Advances in Information Systems, 41 (2010), pp. 27-48. [8] A. Powell, G. Piccoli and B. Ives, "Virtual teams: a review of current literature and directions for future research", The DATABASE for Advances in Information Systems, 35 (2004), pp. 6-36.

[9] J. McAvoy and T. Butler, "The role of project management in ineffective decision making within Agile software development projects", European Journal of Information Systems, 18 (2009), pp. 372-383.

[10] X. Yang, Y. Tong and H. H. Teo, "Fostering Fastresponse Spontaneous Virtual Team: Effects of Member Skill Awareness and Shared Governance on Team Cohesion and Outcomes", Journal of the Association for Information Systems, 16 (2015), pp. 919.

[11] R. E. Quinn, Beyond rational management: Mastering the paradoxes and competing demands of high performance, Jossey-Bass, 1988.

[12] G. T. Fairhurst, W. K. Smith, S. G. Banghart, M. W. Lewis, L. L. Putnam, S. Raisch and J. Schad, "Diverging and converging: Integrative insights on a paradox metaperspective", Academy of Management Annals, 10 (2016), pp. 173-182.

[13] S. Sawyer, P. J. Guinan and J. Cooprider, "Social interactions of information systems development teams: a performance perspective", Information Systems Journal, 20 (2010), pp. 81-107.

[14] J. S.-C. Hsu, T.-H. Chu, T.-C. Lin and C.-F. Lo, "Coping knowledge boundaries between information system and business disciplines: An intellectual capital perspective", Information \& Management, 51 (2014), pp. 283-295.

[15] L. F. Luna-Reyes, J. Zhang, J. R. Gil-García and A. M. Cresswell, "Information systems development as emergent socio-technical change: a practice approach", European Journal of Information Systems, 14 (2005), pp. 93-105. [16] N. Levina, "Collaborating on multiparty ISD projects: A collective reflection-in-action view", Info. Systems Research, 16 (2005), pp. 109-130.

[17] J. Lee, J.-G. Park and S. Lee, "Raising team social capital with knowledge and communication in information systems development projects", International Journal of Project Management, 33 (2015), pp. 797-807.

[18] J. Conklin, Dialogue mapping: Building shared understanding of wicked problems, Wiley, 2005.

[19] E. A. C. Bittner and J. M. Leimeister, "Creating shared understanding in heterogeneous work groups: Why it matters and how to achieve it", Journal of Management Information Systems, 31 (2014), pp. 111-144.

[20] R. O. Briggs, G. L. Kolfschoten and G.-J. d. Vreede, "Toward a theoretical model of consensus building", AMCIS 2005 Proceedings (2005), pp. 12.

[21] A. M. Carton and J. N. Cummings, "A theory of subgroups in work teams", Academy of Management Review, 37 (2012), pp. 441-470.

[22] A. Aggarwal, Decision making in diverse swift teams: An exploratory study, 47th Hawaii International Conference on System Sciences, IEEE, 2014, pp. 278-288.

[23] D. Van Knippenberg and M. C. Schippers, "Work group diversity", Annu. Rev. Psychol., 58 (2007), pp. 515-541.

[24] C. Pflügler, M. Wiesche and H. Krcmar, Subgroups in Agile and Traditional IT Project Teams, 51 st HICSS, 2018. [25] L. Chidambaram, R. P. Bostrom and B. E. Wynne, "A longitudinal study of the impact of group decision support systems on group development", Journal of Management Information Systems, 7 (1990), pp. 7-25.

[26] P. Van den Bossche, W. Gijselaers, M. Segers, G. Woltjer and P. Kirschner, "Team learning: building shared mental models", Instruct. Science, 39 (2011), pp. 283-301. [27] R. K. Yin, Case study research: Design and methods, Sage publications, 1994.

[28] M. B. Miles and A. M. Huberman, Qualitative data analysis: A sourcebook, Sage, Beverly Hills, 1994.

[29] S. McCarthy, P. O'Raghallaigh, C. Fitzgerald and F. Adam, A Typology for Organizational ICT Practice, the 50th Hawaii International Conference on System Sciences, 2017.

[30] J. M. Carroll and P. A. Swatman, "Structured-case: a methodological framework for building theory in information systems research", European Journal of Information Systems, 9 (2000), pp. 235-242.

[31] A. Strauss and J. Corbin, Basics of qualitative research, Newbury Park, CA: Sage, 1990.

[32] T. Parsons, The Social system, Routledge, London, 1951.

[33] P. Bourdieu, Outline of a Theory of Practice, Cambridge university press, 1977.

[34] A. M. Pettigrew, "Context and action in the transformation of the firm", Journal of management studies, 24 (1987), pp. 649-670.

[35] S. McCarthy, P. O'Raghallaigh, C. Fitzgerald and F. Adam, Theorising Antecedents of Cohesion and Conflict in Distributed ISD Project Teams, ICIS 2018 Proceedings, San Francisco, 2018.

[36] E. Miron-Spektor, A. Ingram, J. Keller, W. K. Smith and M. W. Lewis, "Microfoundations of organizational paradox: The problem is how we think about the problem", Academy of Management Journal, 61 (2018), pp. 26-45.

[37] R. L. Wakefield, D. E. Leidner and G. Garrison, "Research note - a model of conflict, leadership, and performance in virtual teams", Information systems research, 19 (2008), pp. 434-455. 